\title{
Model Identification of a Micro Air Vehicle
}

\author{
Jorge Niño ${ }^{1}$, Flavius Mitrache ${ }^{1}$, Peter Cosyn ${ }^{2}$, Robin De Keyser ${ }^{1}$ \\ 1. Department of Electrical Energy, Systems and Automation, Ghent University, \\ Technologiepark 913, 9052 Ghent, Belgium \\ 2. Department of Flow, Heat and Combustion Mechanics, Ghent University, \\ Sint-Pietersnieuwstraat 41, 9000 Ghent, Belgium
}

\begin{abstract}
This paper is focused on the model identification of a Micro Air Vehicle (MAV) in straight steady flight condition. The identification is based on input-output data collected from flight tests using both frequency and time domain techniques. The vehicle is an in-house $40 \mathrm{~cm}$ wingspan airplane. Because of the complex coupled, multivariable and nonlinear dynamics of the aircraft, linear SISO structures for both the lateral and longitudinal models around a reference state were derived. The aim of the identification is to provide models that can be used in future development of control techniques for the MAV.
\end{abstract}

Keywords: Micro Air Vehicles, system identification, flight tests

Copyright (C 2007, Jilin University. Published by Elsevier Limited and Science Press. All rights reserved.

\section{Introduction}

Since 2002 the Department of Flow, Heat and Combustion Mechanics of Ghent University has conducted MAV research. MAVs have been built with 15, 25 and $40 \mathrm{~cm}$ wingspan. The main aim is to build a system of autonomous vehicles for surveillance missions. Before design of autopilots and control systems for navigation, theoretical modeling and system identification by means of flight tests have been applied to the $40 \mathrm{~cm}$ wingspan model (from now on called MAV40).

During recent years, interest in system identification of MAVs has increased. Most of the studies are focused on finding linear models for classic control design. Some authors work with nonlinear identification, trying to find robust models which allow running more accurate simulations, on which advanced control techniques can be applied.

Wu et al.$^{[1]}$ presented an identification experiment applied to a $1 \mathrm{~m}$ wingspan aircraft using time domain techniques, deriving two ARX models. The models are used for control design applying classic methods of compensation. $\mathrm{Hu}$ et al. ${ }^{[2]}$ identified linear models for the dynamics of speeding up process before take-off of small MAV, considering SISO models with drift angle of front wheel as input and heading angle as output. Manaï et al. ${ }^{[3]}$ identified the parameters of the nonlinear model of an MAV using the output-error approach with the aim of replacing an existent gain scheduled PID by a non-linear controller. Tischler ${ }^{[4]}$ used a frequencydomain system identification method by means of a set of tools summarized in a suite called CIFER, for the development and integration of aircraft flight-control systems applied to a rotorcraft. Mettler et al ${ }^{[5]}$ applied CIFER to a model-scale unmanned helicopter, deriving a linear state-space model for its dynamics in hover condition. Salman and Sreenatha ${ }^{[6]}$ used a nonlinear mapping identification method for an MAV, from which they derived a non-linear state space model.

In this paper we present identification results of the MAV40, deriving two linear SISO models, one for the longitudinal dynamics using the elevator deflection as input and the pitch angular rate as output, and the other for lateral dynamics using the aileron deflection as input and the roll angular rate as output.

In section 2 a short description of the MAV40 and 
the hardware and software used in the experiments is given. A summarized theoretical modeling is presented in section $\mathbf{3}$ in order to give an insight into structure and complexity of the system. The frequency domain identification approach, the signals used and the experiment setup are explained in section 4. Results of the identification using time domain technique are presented in section $\mathbf{5}$.

\section{System description}

The MAV40 is a delta-wing aircraft; it has a wingspan of $40 \mathrm{~cm}$, an aspect ratio of 1.8 and a total weight of $252 \mathrm{~g}$ including sensors, actuators and communication systems. Fig. 1 shows the MAV40 during a flight routine and its external components are shown in Fig. 2.

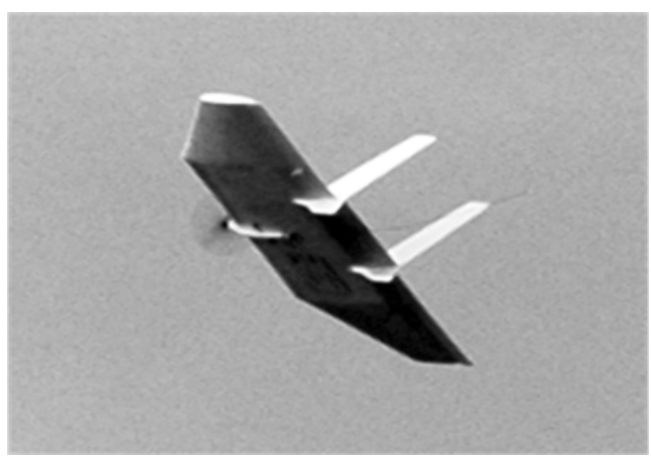

Fig. 1 The UAV40 during a flight test routine.

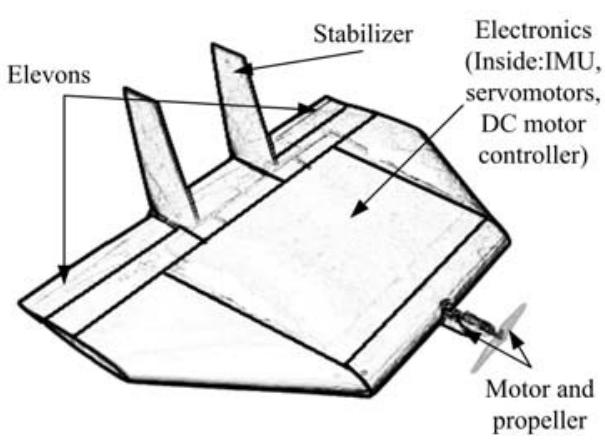

Fig. 2 The $40 \mathrm{~cm}$ wingspan UGent MAV.

The sensor interface is composed of angular rate sensors, accelerometers, pressure sensors, altimeter, GPS system, all of which are integrated in a Inertial Measurement Unit (IMU). The IMU (O-NAVI Phoenix) was programmed using GNU tools for MCORE. The
MCORE family MMC2114 microcontroller is the brain of the embedded system.

A bidirectional communication system MAV-PC was designed and built. This system consists of MaxStream transceivers, RC systems and a grounded PPM reader/generator. The PPM system uses a microchip PIC16F628A and allows permanent communication between the PC and the RC system. In this way it is possible to mix the human pilot signal with the identification signal designed offline in the PC. This is very important because in that way the pilot can find the necessary trim values to put the MAV in a desired flight condition and at the same time the PC can excite the system in the desired range of frequency for identification, reducing risk of crashes.

Acquisition of data was done by means of a connection between the transceiver on the ground and the PC. Software in Visual Basic 6.0 was designed for this aim. Analysis and processing of data were done using Matlab.

\section{MAV mathematical model}

In order to find the frequency band of interest to design the identification signals, the dynamical model of the MAV was analyzed. Klein and Morelli ${ }^{[7]}$ presented a detailed explanation about the dynamic models for aerial vehicles.

The dynamics of an aircraft has a non-linear, multivariable and coupled behavior. However, starting from a steady flight condition two uncoupled linear models that represent closely the behavior of the MAV can be derived. The aim of the identification process is to find those two linear models for use in control design.

\subsection{Inputs}

Regarding the manipulated variables, Fig. 2 shows that the MAV40 has three inputs, two elevons and one electrical propeller. Elevons are deflection surfaces and have a direct influence on the aerodynamic forces. They can behave as elevators or ailerons at the same time, resulting in two different inputs, elevator deflection $\left(\delta_{\mathrm{e}}\right)$ and aileron deflection $\left(\delta_{\mathrm{a}}\right)$, both of them with unit in radian.

Two servomotors act as actuators for the elevons. 
They are modeled as second order systems. The identification procedure explained in section 4 was applied, to obtain the following transfer function

$$
\frac{\delta_{\mathrm{e}}}{\delta_{\text {eref }}}=\frac{\delta_{\mathrm{a}}}{\delta_{\text {aref }}}=\frac{9025}{(s+95)^{2}},
$$

where $\delta_{\text {eref }}$ and $\delta_{\text {aref }}$ are the desired values in radian. The electrical propeller supplies the propulsion force and its input value $\left(\delta_{\mathrm{p}}\right)$ is nondimensional. This value represents the voltage fraction of battery applied to the motor.

\subsection{Reference frames}

The analysis is made considering the earth axes $\left(x_{\mathrm{E}}\right.$, $\left.y_{\mathrm{E}}, z_{\mathrm{E}}\right)$ and the vehicle-carried earth axes $\left(x_{\mathrm{V}}, y_{\mathrm{V}}, z_{\mathrm{V}}\right)$ as inertial frames of reference (see Fig. 3). The body axes $(x, y, z)$ and the wind axes are used as a reference for the inertial forces and aerodynamic forces respectively.

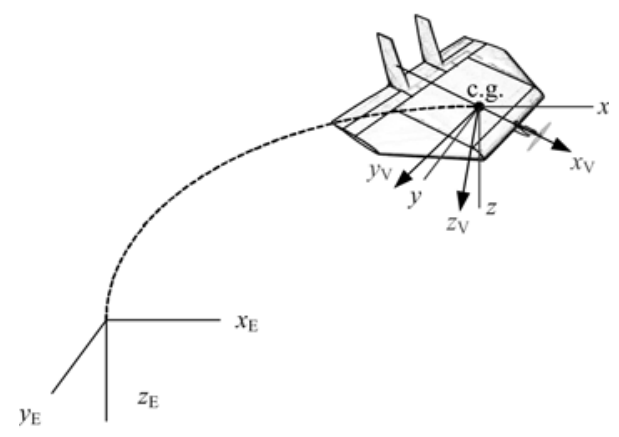

Fig. 3 The reference frames.

\subsection{Motion equations}

The MAV40 dynamics can be described by a nonlinear state-space system with twelve state variables and three inputs. The equations are obtained by applying Newton's second law for rotating axis system for forces and moments, the Euler angles rotational kinematics and the navigation equations.

The motions of the MAV in translational and rotational forms are described by Newton's second law for rotating axis system as

$$
\begin{gathered}
\boldsymbol{F}=m\left[\frac{\mathrm{d} \boldsymbol{V}}{\mathrm{d} t}+(\boldsymbol{\omega} \times \boldsymbol{V})\right], \\
\boldsymbol{M}=\boldsymbol{I} \frac{\mathrm{d} \boldsymbol{\omega}}{\mathrm{d} t}+(\boldsymbol{\omega} \times \boldsymbol{I} \boldsymbol{\omega}),
\end{gathered}
$$

where $\boldsymbol{F}$ is the applied force vector, $m$ is the mass, $\boldsymbol{V}$ is the velocity, $\boldsymbol{M}$ is the applied moment vector about the center of gravity, $\boldsymbol{\omega}$ is the angular velocity vector and $\boldsymbol{I}$ is the inertia matrix. The components of the $\omega$ vector are the roll $(p)$, pitch $(q)$ and yaw $(r)$ angular rates. $V$ has orthogonal components $u, v$ and $w$. Fig. 4 shows the 6DOF system described by Eqs. (2) and (3).

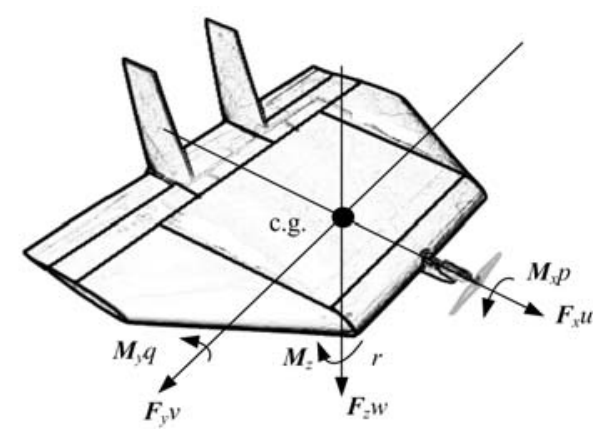

Fig. 4 Body-axes components.

The forces and moments applied are due to gravity, propulsion and aerodynamics. The gravity acting at the c.g. is supposed to be uniform and constant therefore it does not produce a moment.

Since the gravity acts along the $z$-axis of the Earth axis system, it is necessary to introduce the Euler angles in order to express the aircraft orientation with respect to the vehicle-carried earth axes. They are the roll, the pitch and the yaw angle rotation $(\Phi, \theta, \psi)$ around the axis $x_{\mathrm{V}}$, $y_{\mathrm{V}}$ and $z_{\mathrm{V}}$ respectively (see Fig. 5).

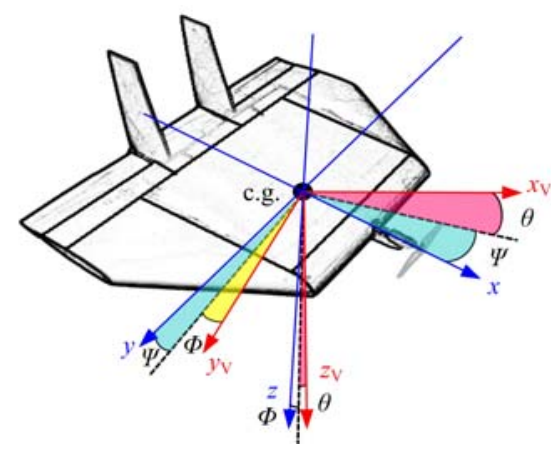

Fig. 5 Euler angles description.

Normally aerodynamic angles (angle of attack $(\alpha)$ and the sideslip angle $(\beta))$ and air speed $(V)$ are used insteading the body axis velocities in order to express the aerodynamic forces. Eqs. (4), (5) and (6) and Fig. 6 
illustrate that relations.

$$
\begin{aligned}
\alpha & =\tan ^{-1}\left(\frac{w}{u}\right), \\
\beta & =\sin ^{-1}\left(\frac{v}{V}\right), \\
V & =\sqrt{\left(u^{2}+v^{2}+w^{2}\right)},
\end{aligned}
$$

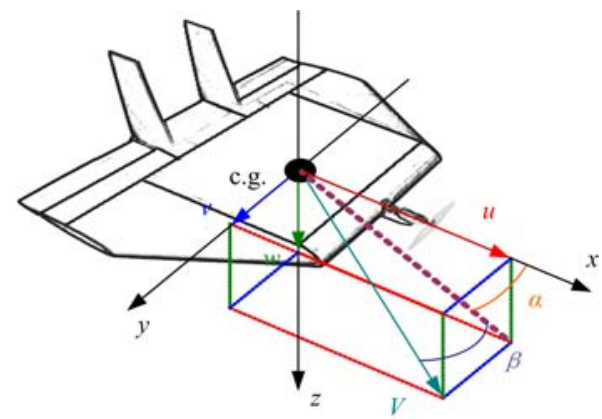

Fig. 6 Aerodynamic angles.

The rotational kinematics is described by the differential equations of the Euler angles as

$$
\begin{aligned}
& \dot{\phi}=p+\tan \theta(q \sin \phi+r \cos \phi), \\
& \dot{\theta}=q \cos \phi-r \sin \phi, \\
& \dot{\psi}=\frac{q \sin \phi+r \cos \phi}{\cos \theta} .
\end{aligned}
$$

The outer loop in a control system for MAV takes into account navigation variables, therefore it is necessary to consider the position with respect to the earth coordinates as

$$
\begin{aligned}
\dot{x}_{\mathrm{E}}= & V \cos \alpha \cos \beta \cos \theta \\
& +V \sin \beta(\cos \psi \sin \theta \sin \phi-\sin \psi \cos \phi) \\
& +V \sin \alpha \cos \beta(\cos \psi \sin \theta \cos \phi+\sin \psi \sin \phi),
\end{aligned}
$$

$$
\begin{aligned}
\dot{y}_{\mathrm{E}}= & V \cos \alpha \cos \beta \sin \theta \\
& +V \sin \beta(\sin \psi \sin \theta \sin \phi+\cos \psi \cos \phi) \\
& +V \sin \alpha \cos \beta(\sin \psi \sin \theta \sin \phi-\cos \psi \sin \phi),
\end{aligned}
$$

$$
\begin{aligned}
\dot{h}=-\dot{z}_{\mathrm{E}}= & V \cos \alpha \cos \beta \sin \theta-V \sin \beta \cos \theta \sin \phi \\
& -V \sin \alpha \cos \beta \cos \theta \cos \phi .
\end{aligned}
$$

\subsection{Nonlinear state-space model}

Deriving the differential equations from Eqs. (2) and (3), and taking Eqs. (7)-(12), a system with highly nonlinear and coupled dynamics is obtained. The detailed model structure and components can be found in Ref. [7], expressing in state space form as

$$
\begin{aligned}
& \dot{\boldsymbol{x}}=f(\boldsymbol{x}, \boldsymbol{u}), \\
& \boldsymbol{x}=\left[\begin{array}{llllllllllll}
V & \alpha & \beta & p & q & r & \varphi & \theta & \psi & x_{\mathrm{E}} & y_{\mathrm{E}} & h
\end{array}\right]^{\mathrm{T}}, \\
& \boldsymbol{u}=\left[\begin{array}{lll}
\delta_{\mathrm{e}} & \delta_{\mathrm{a}} & \delta_{\mathrm{p}}
\end{array}\right]^{\mathrm{T}} .
\end{aligned}
$$

Using the MAV40 physical specifications and CAD simulations, preliminary numerical values for the model parameters have been obtained.

\subsection{Simplification and linearization}

In order to make the structures of the models to be identified, the nonlinear system described above is linearized at the following operation point:

$$
\begin{aligned}
& \boldsymbol{x}_{0}=\left[\begin{array}{llllllll}
8.64 \mathrm{~m} \cdot \mathrm{s}^{-1} & 0.19 \mathrm{rad} & 0 & 0 & 0 & 0 & 0
\end{array}\right. \\
& \left.0.19 \mathrm{rad} \quad 0 \quad 0 \quad 0 \quad 0 \quad h_{0}\right]^{\mathrm{T}} \text {, } \\
& \boldsymbol{u}_{0}=\left[\begin{array}{lll}
0.142 \mathrm{rad} & 0 & 34.28 \%
\end{array}\right]^{\mathrm{T}} \text {. }
\end{aligned}
$$

This operation point represents the straight steady flight condition desired for the MAV40. The state equations for $x_{\mathrm{E}}, y_{\mathrm{E}}, h$ and $\psi$ can be eliminated because those variables do not influence the dynamics of $p$ and $q$, which are the variables of interest. By applying small disturbance theory, the following equations are derived:

$$
\begin{gathered}
\Delta x=\boldsymbol{x}-\boldsymbol{x}_{0}, \\
\Delta \boldsymbol{u}=\boldsymbol{u}-\boldsymbol{u}_{0}, \\
\Delta \dot{\boldsymbol{x}}=\boldsymbol{A} \Delta \boldsymbol{x}+\boldsymbol{B} \Delta \boldsymbol{u} .
\end{gathered}
$$

Fixing $\delta_{\mathrm{p}}$ at $34.28 \%$, matrices $\boldsymbol{A}$ and $\boldsymbol{B}$ are derived, resulting in two uncoupled SISO models for the longitudinal and lateral dynamics

$$
\begin{aligned}
& \left\{\begin{array}{l}
\Delta \boldsymbol{x}_{\text {Lon }}=\left[\begin{array}{llll}
\Delta V & \Delta \alpha & \Delta q & \Delta \theta
\end{array}\right]^{\mathrm{T}} \\
\Delta \boldsymbol{x}_{\text {Lat }}=\left[\begin{array}{llll}
\Delta \beta & \Delta p & \Delta r & \Delta \varphi
\end{array}\right]^{\mathrm{T}}
\end{array},\right. \\
& \Delta \boldsymbol{u}_{\text {Lon }}=\Delta \delta_{\mathrm{e}} \quad \Delta \boldsymbol{u}_{\text {Lat }}=\Delta \delta_{\text {a }}, \\
& \Delta \boldsymbol{y}_{\text {Lon }}=\Delta q \quad \Delta \boldsymbol{y}_{\text {Lat }}=\Delta p,
\end{aligned}
$$

From this model, the following transfer functions are extracted

$$
\frac{\Delta q}{\Delta \delta_{\mathrm{e}}}=\frac{-426.83 s(s+4.52)(s+0.48)}{\left(s^{2}+0.21 s+0.74\right)\left(s^{2}+20.83 s+431.33\right)},
$$




$$
\frac{\Delta p}{\Delta \delta_{\mathrm{a}}}=\frac{362.01(s-0.22)\left(s^{2}+6.25 s+206.18\right)}{(s+11.27)(s+0.14)\left(s^{2}+5.92 s+279.42\right)} .
$$

Eq. (24) describes the longitudinal dynamics of the MAV40 for the given flight condition. It presents two oscillatory modes, the phugoid and the short period ${ }^{[8]}$. Eq. (25) presents the lateral dynamics with two nonoscillatory modes (spiral and roll) and one oscillatory mode (Dutch roll) ${ }^{[8]}$. The dynamics of the servomotors, as shown in Eq. (1) can be neglected.

In order to provide low order models for control design, reduced structures are derived as

$$
\begin{gathered}
\frac{\Delta q}{\Delta \delta_{\mathrm{e}}}=\frac{-4.47 s(s+0.48)}{\left(s^{2}+0.21 s+0.74\right)}, \\
\frac{\Delta p}{\Delta \delta_{\mathrm{a}}}=\frac{267.12(s-0.22)}{(s+0.14)(s+11.27)} .
\end{gathered}
$$

Bode responses of the linearized and reduced models are shown in Fig. 7.

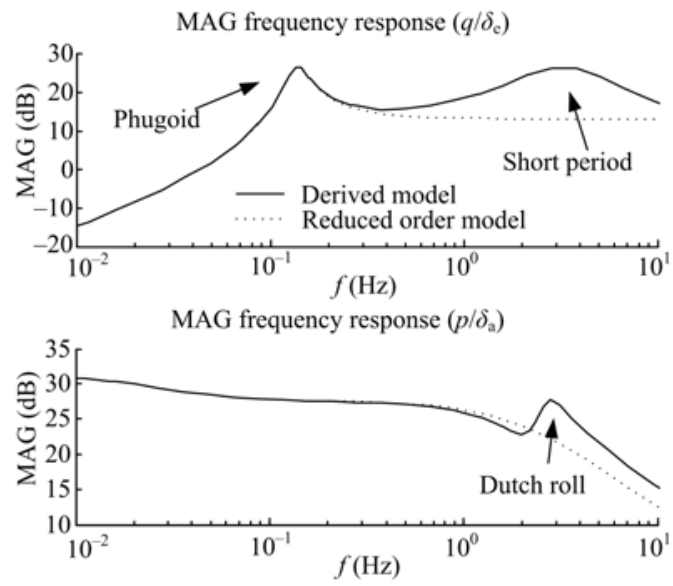

Fig. 7 Theoretical magnitude frequency response for $\Delta q / \Delta \delta_{\mathrm{a}}$ and $\Delta p / \Delta \delta_{\mathrm{e}}$

\section{Identification}

Frequency domain techniques are used to find the valid range of frequency of the captured data, and the initial guesses for the parameter values of the system to be identified. Using that obtained information, time domain techniques are used to find the linear models for use in control design.

CIFER $^{[9]}$ is an integrated set of frequency-domain system-identification programs and utilities. Some of them are used in this work, basically the conditioning of data, performing of FFTs and window combination.

\subsection{Signal design}

Three kinds of signals are used, sine sweep (Fig. 8a) for identification, and doublet (Fig. 8b) and manual input from the pilot (Fig. 8c) for validation. They have been used successfully in identification experiments on MAVs $^{[1,4,7]}$. These signals are added to the trim condition (operation point) which is reached by the pilot. Because large inputs during long period lead the system states going far from the operation point, and small amplitudes reduce the signal to noise ratio, a tradeoff is necessary between frequency range and amplitude during the signal design.

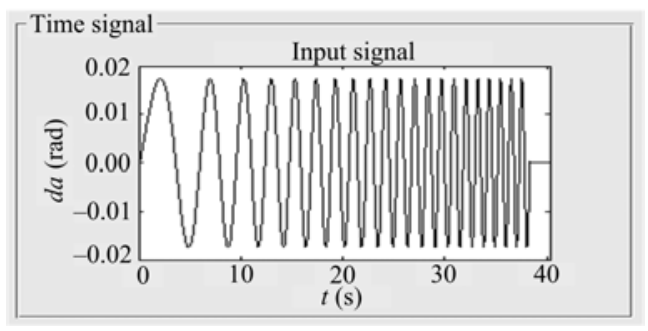

(a) Sine sweep

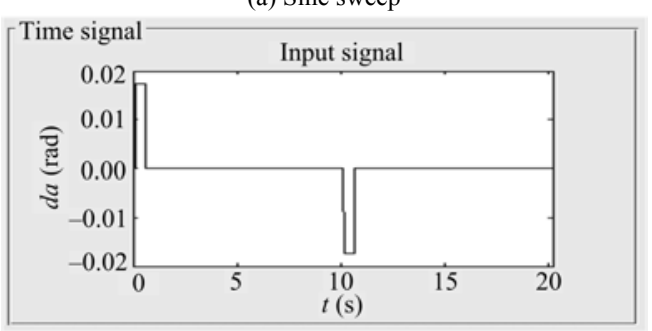

(b) Doublet

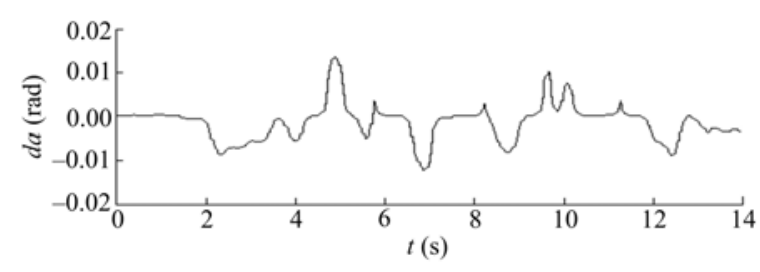

(c) Manual input from the pilot

Fig. 8 Input signals for identification.

In sine sweeps, the frequency sweep inputs are both effective and time efficient and present a flat spectrum, but they must be properly synthesized in order to obtain the desired frequency spectrum. A frequency range from 0.1 to $10 \mathrm{~Hz}$ is used, which covers the dominant dynamics. Manual tests show that maximal amplitudes of 
$6^{\circ}$ in the ailerons, and $5^{\circ}$ in the elevators around the trim condition keep the MAV40 in the linear zone.

In doublets, symmetrical impulses with a width of $0.5 \mathrm{~s}$ are applied in order to evaluate the transient response. Longer impulses bring the system far from the operation point and shorter impulses produce a poor signal to noise ratio in the output.

\subsection{Experiment setup}

The pilot made some flights alone in order to check and bring the system in a stable flight. Once the trim conditions are reached, the identification signal is applied while the aircraft is flying at straight steady condition.

This part requires continuous communication between the pilot and the person who monitors the ground station. Fig. 9 illustrates the situation.

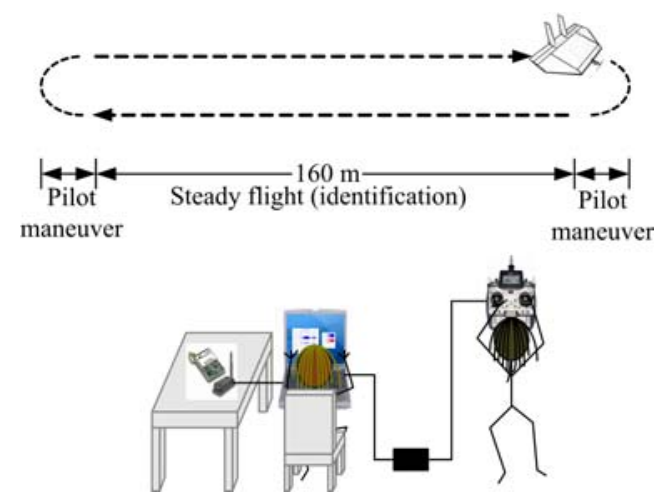

Fig. 9 Flight test routine.

\subsection{Data acquisition}

The captured data contains the information from the real flight condition. The sensors on board include three-axis gyroscopes, three-axis accelerometers, one dynamic pressure sensor and a GPS system. The variables $p, q$ and $r$ are sensed directly. Euler angles can be estimated by means of measurements from the accelerometers and gyroscopes using an extended Kalman filter ${ }^{[10]}$. Airspeed is estimated from measurements using the dynamic pressure sensor. Position can be obtained from the GPS system. The sample time is $23 \mathrm{~ms}$, which is actually the data rate of the $\mathrm{R} / \mathrm{C}$ system. Fig. 10 shows the way for getting information from the twelve states. For this specific case of identification, only the data from $p$ and $q$ are used, but the data from the other sensors are also acquired in order to monitor the system in real time and ensure that the MAV40 is flying at the desired conditions.

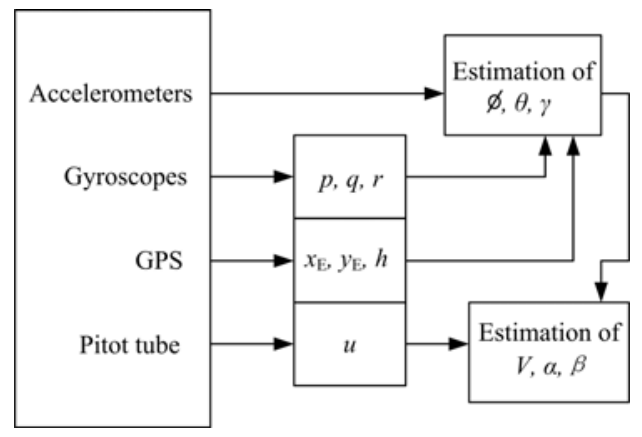

Fig. 10 Measurement and estimation of the twelve states.

\subsection{Data processing - frequency response}

Input and output signals from raw data are previously filtered and bias corrected. Frequency-response of this preprocessed time-domain data is determined by means of the Chirp-z transform ${ }^{[11]}$

$$
X(k)=\sum_{n=0}^{N-1} x(n)\left\{e^{j 2 \pi T_{s}\left[f_{\min }+k\left(f_{\max }-f_{\min }\right) / M\right]}\right\}^{-N},
$$

where $N$ is the number of time samples, $f_{\min }$ and $f_{\max }$ the minimal and maximal frequencies of the range of interest, $T_{S}$ the sample time, $M$ the resolution or number of desired frequency points, and $k$ the index of the Fourier coefficients $(k=0,1, \ldots M-1)$. Using $X$ as the input and $Y$ analogically as the output, the rough estimates of the input autospectrum, output autospectrum and cross spectrum are given by

$$
\begin{aligned}
\widetilde{G}_{x x}(k) & =\frac{2}{(N-1) T_{s}}|X(k)|^{2}, \\
\widetilde{G}_{y y}(k) & =\frac{2}{(N-1) T_{s}}|Y(k)|^{2}, \\
\widetilde{G}_{x y}(k) & =\frac{2}{(N-1) T_{s}}\left|X^{*}(k) Y(k)\right|^{2} .
\end{aligned}
$$

In order to reduce the level of random errors in the spectral analysis, overlapping windowing ${ }^{[9]}$ is applied. Five sizes of windows are used obtaining the same number of smoothed spectral functions. Composite of these functions made in this way is a tradeoff between noise reduction and broad dynamic range. The $i$ th window size is given by 


$$
\widehat{G}_{x x}(k)_{i}=\frac{1}{U n_{r, i}} \sum_{w=1}^{n_{r i}} \widetilde{G}_{x x, w}(k),
$$

where $U$ is the window factor, which is 0.612 for a Hanning window ${ }^{[9]}, n_{r, i}$ is the number of windows for each frequency response, which is given by

$$
n_{r, i}=1+\left[\frac{(N-1)\left(T_{s} / T_{W, i}\right)-1}{1-W_{o v}}\right],
$$

where $W_{o v}$ is the overlap fraction, which is 0.8 , and $T_{W, i}$ is the size of each window in seconds, which is given by

$$
\begin{gathered}
T_{W, i}=0.05 T_{s}(N-1)(i-1)+5(5-i) / f_{\max } \\
i=1,2,3,4,5 .
\end{gathered}
$$

Calculation of the coherence function is made for the five frequency responses

$$
\hat{\gamma}_{x y}(k)_{i}=\frac{\left|\widehat{G}_{x y}(k)_{i}\right|}{\sqrt{\left|\widehat{G}_{x x}(k)_{i}\right|\left|\widehat{G}_{y y}(k)_{i}\right|}} .
$$

The coherence function gives an idea about the fraction of output spectrum that is linearly correlated to the input spectrum.

Estimation of the normalized random error $\varepsilon_{\mathrm{r}}$ in the magnitude of the frequency-response identification can be calculated with the following equation ${ }^{[9]}$

$$
\left(\varepsilon_{r}\right)_{i}=\frac{\left[1-\hat{\gamma}_{x y}^{2}(k)_{i}\right]^{1 / 2}}{\left|\hat{\gamma}_{x y}(k)_{i}\right| \sqrt{2(N-1)\left(T_{s} / T_{W, i}\right)}} .
$$

Finally, composite of the smoothed spectral functions is made by means of averaging, using a weighting function

$$
G_{x x}(k)=\frac{\sum_{i=1}^{5} W_{i}^{2} \hat{G}_{x x}(k)_{i}}{\sum_{i=1}^{5} W_{i}^{2}}, \text { where } W_{i}=\left[\frac{\left(\varepsilon_{r}\right)_{i}}{\left(\varepsilon_{r}\right)_{\min }}\right]^{-4} .
$$

Similar equations are obtained for $G_{x y}$ and $G_{y y}$. The frequency-response function is given by

$$
H(k)=\frac{G_{x y}(k)}{G_{x x}(k)} .
$$

The procedure explained above was applied to the acquired data from MAV40 flight tests to show the un- coupled dynamics at steady flight condition and give the initial guesses for the models to be identified. Data from elevators, ailerons, pitch rate and roll rate were acquired.

Fig. 11 shows a high coherence for the response $\Delta q / \Delta \delta_{\mathrm{e}}$ below $2 \mathrm{~Hz}$, therefore a linear model for this frequency range can be identified with the captured data. Magnitude and phase plots suggest a system with a negative static gain of $15.74 \mathrm{~dB}$.

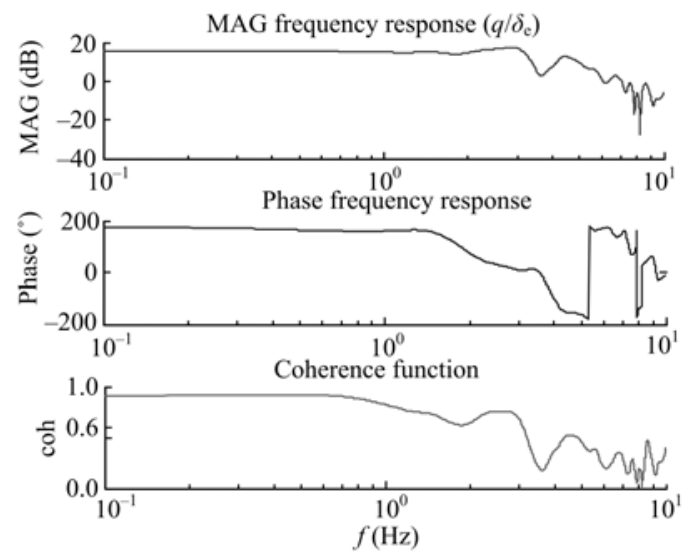

Fig. 11 Frequency response for $\Delta q / \Delta \delta_{\mathrm{e}}$.

Response $\Delta p / \Delta \delta_{\mathrm{a}}$, as shown in Fig. 12, presents an acceptable coherence below $1.5 \mathrm{~Hz}$. Low order models for this range are identified. Initial guess of $17.6 \mathrm{~dB}$ in the static gain is considered for model identification.
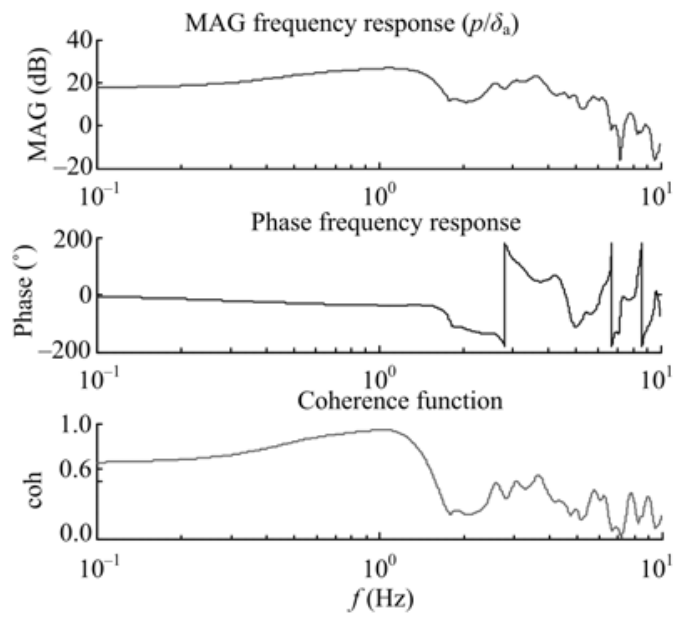

Fig. 12 Frequency response for $\Delta p / \Delta \delta_{\mathrm{a}}$.

Fig. 13 shows the poor coherence for the responses $\Delta q / \Delta \delta_{\mathrm{a}}$ and $\Delta p / \Delta \delta_{\mathrm{e}}$, which confirm the assumption of uncoupling between the longitudinal and lateral dynamics at straight steady flight condition. 

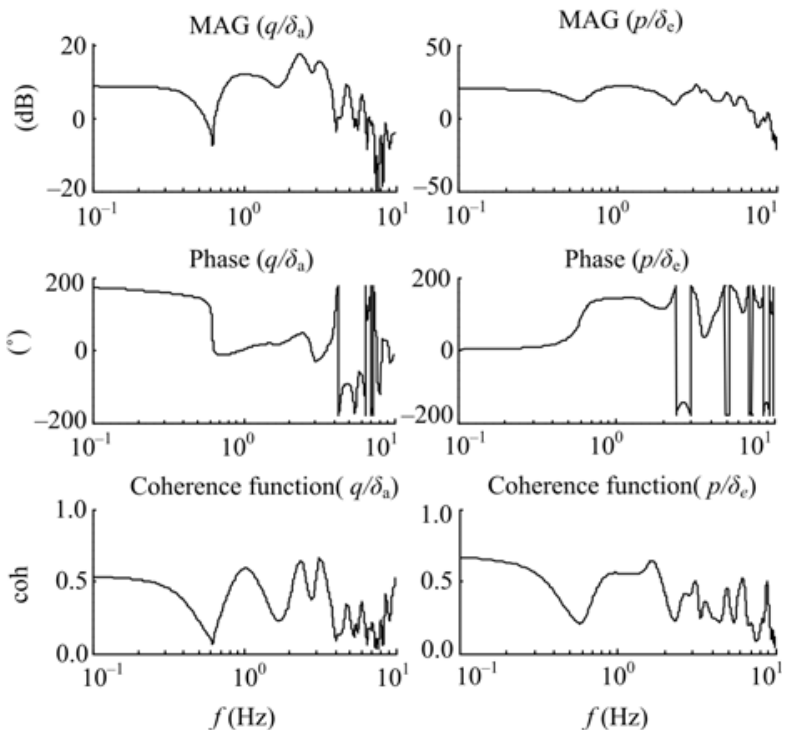

Fig. 13 Frequency response for $\Delta q / \Delta \delta_{\mathrm{a}}$ and $\Delta p / \Delta \delta_{\mathrm{e}}$.

\subsection{Data processing - model identification}

Aided by the previous obtained information, time domain identification techniques are applied. Sets of input/output data for identification and validation are selected from the experiments with the highest coherence function.

Considering white noise in the output (e.g. wind disturbances) the equivalent polynomial structure for the phugoid dynamics in Eq. (26) is given by

$$
y(k)=\frac{B(q)}{F(q)} u(k)+e(k),
$$

which corresponds to an OE (Output Error) model with $n b=3, n f=2, n k=0$. Different structures were used but the one given by Eq. (39) presents the best balance between fit of validation data and simplicity of model.

An equivalent state-space model is also sought with the N4SID algorithm using the Identification Toolbox of Matlab $^{\circledR}$.

\section{Results}

Fig. 14 shows the validation results, and the obtained models are represented by the transfer functions in Eqs. (40) and (41). The magnitude frequency response for the obtained models is shown in Fig. 15.

$$
\begin{aligned}
& \frac{\Delta q}{\Delta \delta_{\mathrm{e}}}(\mathrm{OE})=\frac{-5.7\left(s^{2}+1.75 s+72.18\right)}{\left(s^{2}+0.66 s^{+78.99}\right)}, \\
& \frac{\Delta q}{\Delta \delta_{\mathrm{e}}}(\mathrm{N} 4 \mathrm{SID})=\frac{-1185.56}{\left(s^{2}+11.74 s+198.8\right)} .
\end{aligned}
$$

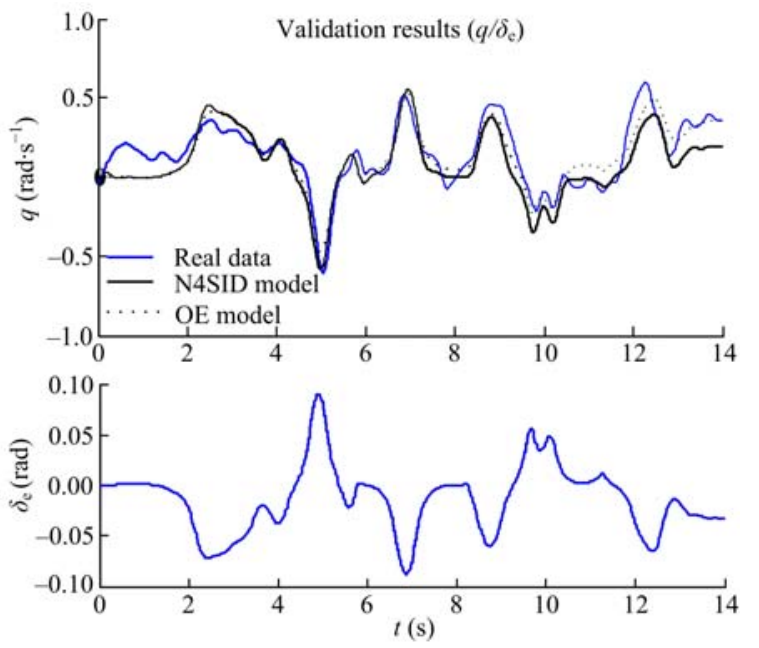

Fig. 14 Validation results for $\Delta q / \Delta \delta_{\mathrm{e}}$ (time domain).

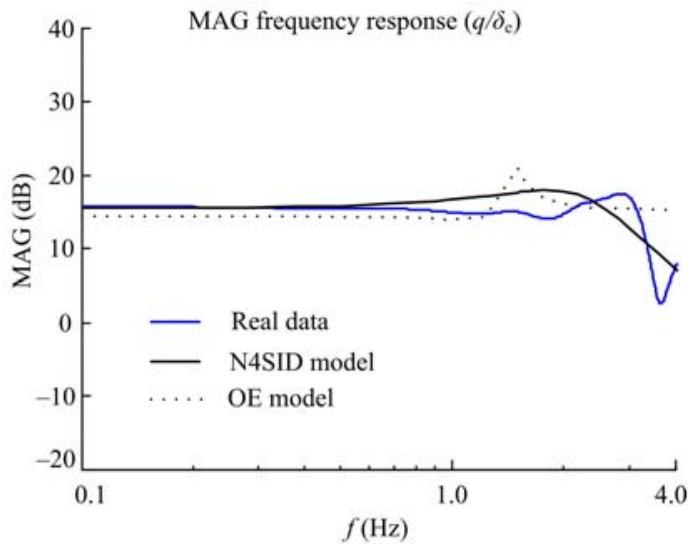

Fig. 15 Validation results for $\Delta q / \Delta \delta_{\mathrm{e}}$ (frequency domain).

The OE model corresponding to the structure of Eq. (27) presents $n b=2, n f=2, n k=1$. The identified transfer function is given by

$$
\frac{\Delta p}{\Delta \delta_{\mathrm{a}}}(\mathrm{OE})=\frac{20.89(s+34.76)}{\left(s^{2}+5.94 s+49.71\right)} .
$$

The N4SID algorithm was also used to find a low order model for the lateral dynamics. The equivalent transfer function of the obtained model is given by 


$$
\frac{\Delta p}{\Delta \delta_{\mathrm{a}}}(\mathrm{N} 4 \mathrm{SID})=\frac{-23.12(s-35.62)}{\left(s^{2}+3.95 s+68.59\right)} .
$$

Validation results for the lateral model in time domain are shown in Fig. 16. The validation in the frequency response is shown in Fig. 17.
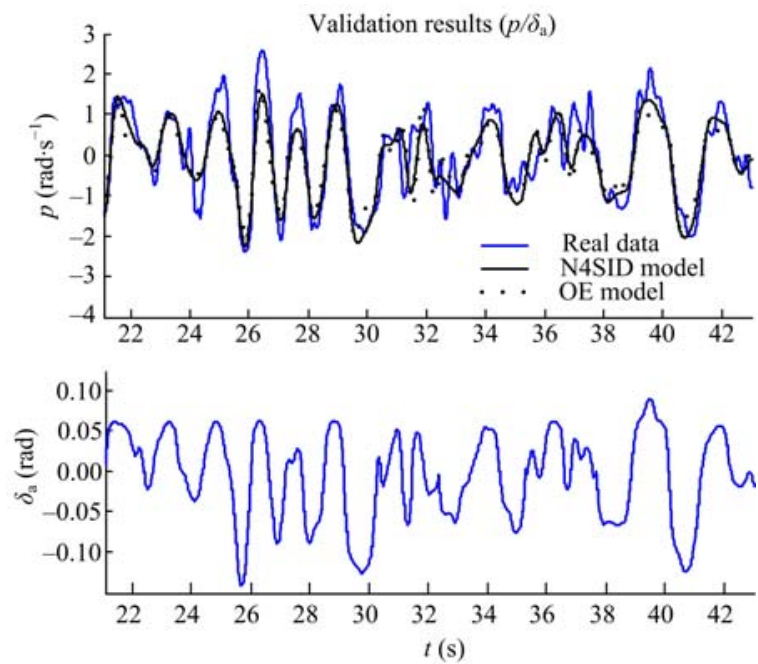

Fig. 16 Validation results for $\Delta p / \Delta \delta_{\mathrm{a}}$ (time domain).

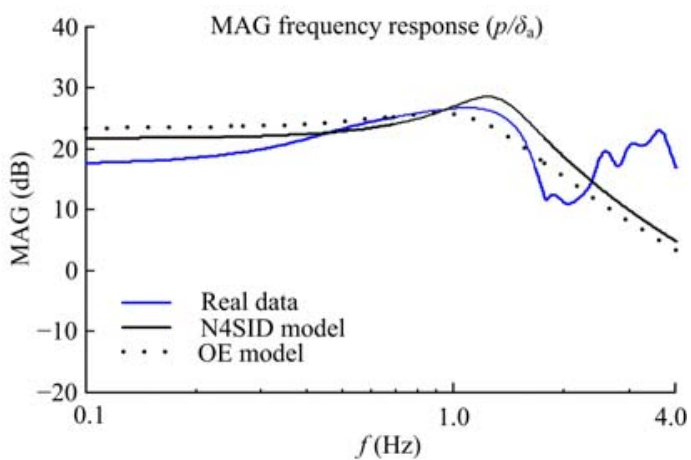

Fig. 17 Validation results for $\Delta p / \Delta \delta_{\mathrm{a}}$ (frequency domain).

Data fits well for the range of frequency of the study, which corresponds to the spectral region where the dynamics that we are interested in controlling is located.

\section{Conclusions and future steps}

Modeling and identification of a $40 \mathrm{~cm}$ wingspan MAV were presented. Theoretical modeling was done in order to gain an initial insight into the complexity of the system, coupling properties, and linearity. Based on that information, identification experiments were done, obtaining a set of SISO models, of which two models for longitudinal and lateral dynamics were presented.

Frequency domain techniques were applied in order to obtain some prior information for the models to be identified, and show experimentally the uncoupled characteristic of the MAV40 dynamics under straight steady flight condition.

Applying time domain techniques, two kinds of models were identified. Using the prediction error method, OE models were found, and using the algorithm N4SID, state space models were derived.

Validation of the identified models presents good results in the frequency region of interest, and can be used for control design purposes.

On a set of SISO systems, classic control strategies (PI, PID controllers) as well as more advanced control algorithms (in this case the EPSAC algorithm for SISO systems) have been applied for performance comparison.

The next step is reconsidering the MIMO system for the MAV40 model and applying the EPSAC algorithm for multivariable systems. So the results when testing this controller on the MAV40 should have better accuracy than in the case of considering the MIMO model divided in SISO models. Design and results details will be present in the future.

\section{Acknowledgement}

J. Niño gratefully acknowledges the financial support of EU ALFA Labioproc Project (Project II-0407-FA) and Universidad de Ibagué (Colombia). The MAV research in Ghent University is supported by the Institute for the Promotion of Innovation by Science and Technology in Flanders (IWT/OZM/060459) and the Industrial Research Fund of the Ghent University.

\section{References}

[1] Wu H Y, Sun D, Zhou Z Y. Model identification of a micro air vehicle in loitering flight based on attitude performance evaluation. IEEE Transactions on Robotics, 2004, 20, 702-712.

[2] Hu C H, Huang X L, Hu J C, Zhu J H. System identification of a small UAV's speeding up process before take-off. 5th Asian Control Conference, 2004, 1, 392-395. 
[3] Manaï M, Desbiens A, Gagnon E. Identification of a UAV and design of a hardware-in-the-loop system for nonlinear control purposes. AIAA Guidance, Navigation, and Control Conference and Exhibit, Unmanned Systems: Flight Control III, San Francisco, USA, 2005, 6483-6488.

[4] Tischler M B. System identification methods for aircraft flight control development and validation. Advances in Aircraft Flight Control, Taylor and Francis, London, 1996.

[5] Mettler B, Tischler M B, Kanade T. System identification of small-size unmanned helicopter dynamics. American Helicopter Society 55th Forum, Montreal, Canada, 1999, 1706-1717.

[6] Salman S A, Sreenatha A G. Nonlinear identification of unmanned aircraft vehicle. International Conference on
Automatic Control and System Engineering, Cairo, Egypt, 2005, 23-27.

[7] Klein V, Morelli E A. Aircraft System Identification: Theory and Practice, AIAA, Blacksburg, USA, 2006.

[8] Nelson R C. Flight Stability and Automatic Control, McGraw-Hill Book Company, New York, USA, 1989.

[9] Tischler M B, Remple R K. Aircraft and Rotorcraft System Identification: Engineering Methods with Flight-Test Examples, AIAA, Blacksburg, USA, 2006.

[10] Marmion M. Airborne Attitude Estimation Using a Kalman Filter, Master thesis, Longyearbyen, Norway, 2006.

[11] Rabiner L. The chirp z-transform algorithm - a lesson in serendipity. IEEE Signal Processing Magazine, 2004, 21, $118-119$. 